\title{
Podocalyxin-like and RNA-binding motif protein 3 are prognostic biomarkers in urothelial bladder cancer: a validatory study
}

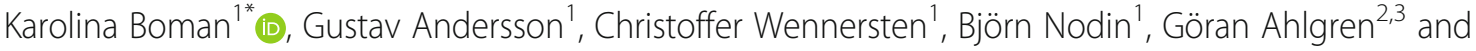
Karin Jirström ${ }^{1}$

\begin{abstract}
Background: Urothelial bladder cancer (UBC) is a disease that often is discovered when the tumour is non-muscle invasive, i.e. in Ta or T1 stage. Some patients will progress into muscle-invasive disease, a potentially deadly condition. Although there are some prognostic models, the need for prognostic and predictive biomarkers is considerate and urgent. Membranous expression of podocalyxin-like protein 1 (PODXL) and low expression of the RNA-binding motif 3 (RBM3) has previously been shown to be associated with an aggressive tumour phenotype and poor prognosis in several forms of cancer, including UBC. In this study, we sought to validate the prognostic impact of PODXL and RBM3 in an independent cohort of UBC.
\end{abstract}

Methods: Using tissue microarrays and immunohistochemistry, PODXL and RBM3 expression was evaluated in 272 incident UBC cases from the prospective, population-based cohort study Malmö Diet and Cancer. Kaplan-Meier analysis and Cox proportional hazards modelling were used to evaluate the prognostic impact of these markers on 5-year overall survival (OS).

Results: In line with previous studies, both membranous PODXL expression and low RBM3 expression was significantly associated with disadvantageous clinicopathological features. Membranous PODXL expression was significantly associated with a reduced 5-year overall survival in the entire cohort (univariable HR 3.28; 95\% Cl 1.89-5.69), but this association did not remain significant in multivariable analysis. In T1 tumours, PODXL was significantly associated with reduced survival in univariable analysis ( $\mathrm{HR}=2.83 ; 95 \% \mathrm{Cl} 1.04-7.72)$ and borderline significant in multivariable analysis $(\mathrm{HR}=2.60 ; 95 \% \mathrm{Cl}$ 0.91-7.39). Low RBM3 expression was an independent predictor of a reduced survival in the entire cohort (univariable HR 3.19; $95 \% \mathrm{Cl} 2.02-5.04$, and multivariable HR 1.85; 95\% Cl 1.11-3.09), and in T1 tumours (univariable HR 2.64; 95\% Cl 1.11-6.27, and multivariable HR 2.63; 95\% Cl 1.01-6.84).

Conclusions: A link between membranous PODXL expression and clinically more aggressive tumours was further confirmed, but PODXL expression was not an independent prognostic biomarker in this study. Low RBM3 expression was validated as an independent factor of poor prognosis in $\mathrm{UBC}$, including $\mathrm{T} 1$ disease. These findings suggest that these biomarkers could be useful in stratifying patients with non-muscle invasive disease for more aggressive first line treatment.

Keywords: PODXL, RBM3, Bladder cancer, Prognosis

\footnotetext{
* Correspondence: karolina.boman@med.lu.se

'Department of Clinical Sciences Lund, Division of Oncology and Pathology,

Lund University, Lund, Sweden

Full list of author information is available at the end of the article
} 


\section{Background}

The majority of patients with urothelial bladder cancer (UBC) have Ta and T1 tumours, i.e. non-muscle invasive (NMI) disease, at diagnosis [1]. However, the unpredictable behaviour of these tumours regarding recurrence and progression into muscle-invasive disease makes treatment decisions difficult. NMI lesions are typically managed with transurethral resection, and selectively with intravesical therapy [2]. Patients with T1 disease, particularly those with high-risk features, are at risk of disease progression and may benefit from additional therapy. In a recent study of a large cohort of patients with non-muscle invasive UBC, the progression rates in T1G3 disease were around 20\%, despite local treatment with Bacillus Calmette Guerin (BCG) [3]. Patients who progress into muscle-invasive disease (T2-T4) have a $50 \%$ risk of developing distant metastasis, even with potentially curative surgery [4]. Moreover, patients with T1 disease that progresses into MI disease seem to have a worse prognosis than those who present as primary MI $[5,6]$. Surgery via radical cystectomy and pelvic lymph node dissection, with or without neoadjuvant therapy, remain the gold standard for potentially curative T2-T4 tumours. The survival rates 5 years after surgery span between 25 and $80 \%$, being worse with higher pT stage and the presence of lymph node metastasis [4].

Hence, there is a vast and urgent need to find predictive biomarkers to help clinicians identify the patients with non-invasive tumours in need of more aggressive first line treatment, since accurate prediction of the risk of progression into muscle-invasive disease could be the key to improving patient survival in the non-muscle invasive tumour group.

Podocalyxin-like protein 1 (PODXL) is a protein involved in cell adhesion and morphology [7, 8]. Strong, in particular membranous, expression has been found to correlate with more aggressive tumours and poor survival in many cancer forms such as breast cancer [9], colorectal cancer [10-12], ovarian cancer [13], glioblastoma [14] as well as UBC [15]. In the latter study, we showed that PODXL is an independent risk factor for progressive disease and death in patients with all T-stages of UBC, as well as in the Ta/T1 subgroup.

RNA-binding motif protein 3 (RBM3) is an RNA and DNA binding protein that has previously been shown to be upregulated in cancer tissues compared with normal tissue [16], but to be associated with favourable prognosis in several major cancer forms such as breast, ovarian, prostate, testicular, esophageal, colorectal cancer and malignant melanoma [17-25]. In a previous study, we examined the prognostic value of RBM3 expression in a large group of patients with UBC $(n=343)$, and found reduced expression to be associated with clinically more aggressive tumours and an independent factor of poor prognosis in the cohort as a whole, as well as in the Ta/ T1 group [26].

The aim of this study was to validate the clinicopathological correlates and prognostic impact of PODXL and RBM3 expression, respectively, in an independent cohort of UBC.

\section{Methods}

\section{Study group}

The study encompasses tumours from incident cases of UBC in the Malmö Diet and Cancer study (MDCS). The MDCS is a prospective population-based study, primarily aimed at examining the impact of a Western diet, low in fruit and vegetables and high in fat, on the risk of certain types of cancer. The baseline examinations took place between 1991 and 1996 in Malmö, Sweden. The source population invited to participate was comprised by women born between 1923 and 1950 and men born between 1923 and 1945 (74,138 persons). The cohort and the recruitment procedures have been published elsewhere [27]. Participants completed a detailed questionnaire, anthropometric measurements and a dietary assessment. A total of 28,098 participants completed all baseline examinations. The Ethical Committee at Lund University approved the MDCS. At the end of follow-up, December 31 2010, a total of 355 cases of incident urothelial cancer (UC) were recorded, of which 335 (95,7\%) were located to the bladder. All cases of UC were histopathologically re-evaluated by a board certified pathologist according to the WHO grading system of 2004. The cohort has been described in detail previously [28] The mean and median follow-up time was 6.25 and 5.04 years, respectively (range $0.11-20.69$ ).

\section{TMA construction and immunohistochemistry}

Tissue microarrays (TMAs) were constructed using a semi-automated arraying device (TMArrayer, Pathology Devices, Westminster, MD, USA). All tumour samples were represented in duplicate tissue cores of $1 \mathrm{~mm}$. Staining of both RBM3 and PODXL was evaluated by two independent observers (KJ and $\mathrm{KB}$ ) who were blinded to clinical and outcome data. Scoring differences were discussed in order to reach consensus. A total number of 272 tumours, of which 264 (97.1\%) were located to the bladder, were eligible for TMA construction. The cases excluded from TMA-construction had either only cytology or autopsy specimens available, or an insufficient amount of tumour tissue in the biopsies. A comparison of the TMA cohort and non-TMA cohort is provided in [28].

For immunohistochemical analysis, 4- $\mu \mathrm{m}$ TMA sections were automatically pre-treated using the PT Link system and then stained in an Autostainer Plus (Dako, Glostrup, Denmark). For evaluation of PODXL expression, the antibody AMAb90667, Atlas Antibodies AB, 
Stockholm, Sweden, diluted 1:250, was used. The expression of PODXL was recorded as negative (0), weak cytoplasmic positivity in any proportion of cells (1), moderate cytoplasmic positivity in any proportion (2), distinct membranous positivity in $\leq 50 \%$ of cells (3) and distinct membranous positivity in $>50 \%$ of cells, as previously described $[10,11,15]$.

For evaluation of RBM3 expression, the mouse monoclonal anti-RBM3 antibody AMAb90655, Atlas Antibodies AB, Stockholm, Sweden, diluted 1:150 was used. RBM3 expression was mainly expressed in the nuclei and the fraction of cells with nuclear positivity (NF) denoted as $0(0-1 \%), 1(2-25 \%), 2(26-75 \%)$ and $3(>75 \%)$, and the intensity of the staining ((NI) as 0 (negative), 1 (moderate) and 2 (strong). A combined nuclear score (NS), e.g. multiplier of NF $x \mathrm{NI}$, was then constructed as previously described $[18,19,26]$.

\section{Statistical methods}

Chi-square test was used for comparison of biomarker expression with patient and tumour characteristics. Kaplan-Meier analysis and log-rank test were applied to illustrate differences in survival between different strata of RBM3 and PODXL expression. Cox regression proportional hazards models were used to estimate the impact of RBM3 and PODXL expression on 5-year overall survival (OS) in both univariable and multivariable analysis, adjusted for age at diagnosis (continuous), sex, Tstage and tumour grade. Classification and regression tree (CRT) analysis was applied to find an appropriate cut off value for RBM3 expression.

All calculations were performed using IBM SPSS Statistics for Mac version 23.0 (IBM, Armonk, NY, USA). All statistical tests were two-sided and a $P$-value $<0.05$ was considered statistically significant.

\section{Results}

\section{Distribution of PODXL and RBM3 expression}

After antibody staining and optimization, PODXL expression could be evaluated in 262/264 (99.2\%) tumours. Membranous PODXL staining was denoted in 27/262 cases $(10.3 \%)$ and there was no obvious heterogeneity between duplicate cores.

RBM3 expression could be evaluated in 259/264 (98.1\%) tumours, whereby negative RBM3 staining (NS $=0)$ was denoted in $26(10.0 \%)$ cases, intermediate staining in 78 (30.1\%) cases and strong staining in $>75 \%$ of the cells in 155 (59.8\%) cases. Interestingly, all cases with NS 6 or 9, i.e. tumours denoted as being RBM3 high, were homogenously so. The tumours with lower expression (NS 0-4) had some heterogeneity between cores, but remained in the low RBM3 category. The "worst nuclear score" from each tumour was chosen for further analysis.
Sample images of the different PODXL and RBM3 stainings are shown in Figs. 1 and 2, respectively.

\section{Associations of PODXL and RBM3 expression with clinicopathological factors}

The associations of PODXL and RBM3 expression, respectively, with patient and tumour characteristics are shown in Table 1.

Membranous PODXL expression was significantly associated with higher T-stage and high-grade tumours $(p<$ 0.001 for both). High RBM3 expression was strongly and significantly associated with lower T-stage and low-grade tumours ( $p=0.001$ for both).

None of the investigated biomarkers were significantly associated with sex, or age at diagnosis.

\section{Association between PODXL expression and survival}

The expression of PODXL was dichotomized into membranous and non-membranous expression in line with previous studies $[11,15]$.

As shown in Fig. 3, Kaplan-Meier analysis of the entire cohort demonstrated a significant association between membranous PODXL and a shorter 5-year OS in the entire cohort $(p<0.001$, Fig. 3a), and in T1 disease $(p<0.001$, Fig. 3b). PODXL was not prognostic in T2-T4 disease (Fig. 3c). Notably, no membranous PODXL expression was seen in Ta tumours.

As shown in Table 2, univariable Cox regression analysis confirmed the significant association between membranous PODXL expression and a reduced 5-year OS in the entire cohort (HR 3.28; 95\% CI 1.89-5.69) and in T1 tumours (HR 2.83; 95\% CI 1.04-7.72). These associations did however not remain significant in multivariable analysis (Table 2).

\section{Association between RBM3 expression and survival}

Kaplan-Meier analysis of different categories of RBM3 expression (NS) revealed a stepwise reduced 5 year overall survival (OS), with the shortest survival for RBM3 negative tumours and the longest survival for the two highest categories, i.e. NS 6 and 9 (Additional file 1). CRT analysis confirmed these associations (Additional file 2). Hence, for statistical purposes, categories of the expression of RBM3 were dichotomized into low (NS = $0-4)$, and high (NS $=6-9$ ).

Kaplan Meier analysis revealed that cases with low RBM3 expression had a significantly shorter 5 -year OS than cases with high expression $(p<0.001$, Fig. $4 a)$. There was no significant association between RBM3 expression and survival in Ta tumours (data not shown), but in T1 tumours, low RBM3 expression was significantly associated with a reduced 5-year OS ( $p=0.023$, Fig. $4 \mathrm{~b})$. In muscle-invasive (T2-T4) tumours, there was a borderline significant trend 

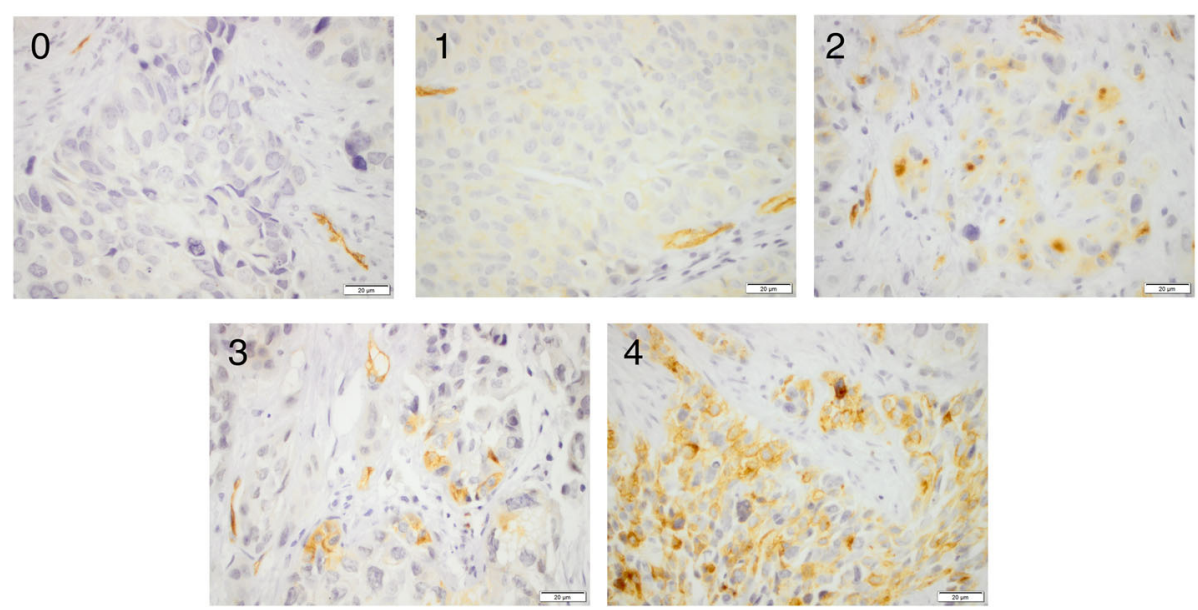

Fig. 1 Sample immunohistochemical images of PODXL staining. Images (40x magnification) representing all different scores from 0 to 4 , with 3 and 4 denoting membranous expression in $<=50 \%$ or $>50 \%$ of tumour cells, respectively. Of note, score 2 represents a strong "dot-like" cytoplasming staining

towards a reduced 5-year OS for tumours with low RBM3 expression ( $p=0.061$, Fig. 4c).

As shown in Table 2, univariable Cox regression analysis confirmed the significant association between low RBM3 expression and a shorter 5 -year OS in the entire cohort (HR 3.19; 95\% CI 2.02-5.02) and in T1 tumours (HR 2.64; 95\% CI 1.11-6.27). In multivariable analysis, adjusted for age, sex, T-stage and grade, RBM3 remained an independent prognostic factor in the entire cohort (HR 1.85; 95\% CI 1.11-3.09) and in T1 tumours (HR 2.63 95\% CI 1.01-6.84). In T2-T4 tumours, none of the established clinicopathological factors remained significant, but RBM3 was borderline prognostic in both univariable (HR 2.01, 95\% CI 0.95-4.23) and multivariable analysis (HR 2.22, 95\% CI 0.94-5.28).
When both PODXL and RBM3 were included in the multivariable model, only low RBM3 expression and Tstage remained significant prognostic factors in the entire cohort (HR 1.97; 95\% CI 1.18-3.31 and HR 4.88; 95\% CI 2.15-11.09, respectively). In the $\mathrm{T} 1$ subgroup, only RBM3 showed a significant association with survival (HR 2.79; 95\% CI 1.05-7.36). In the T2-T4 group, none of the investigated biomarkers remained significant, although RBM3 was borderline so (HR 2.35; 95\% CI 0.97-5.72).

PODXL and RBM3 expression in relation to tumour type The re-classified tumours were also divided into four categories according to tumour type, i.e. non-invasive low grade $(n=98)$, non-invasive high grade $(n=16)$, classic invasive $(n=99)$ and invasive non-classic $(n=$


Fig. 2 Sample immunohistochemical images of RBM3 staining. Images (20x magnification) representing different nuclear scores of RBM3 staining, i.e. fraction $\times$ intensity 
Table 1 Associations between RBM3 and PODXL expression and clinicopathological characteristics

\begin{tabular}{|c|c|c|c|c|c|c|}
\hline \multicolumn{4}{|c|}{ PODXL Expression $(n=262)$} & \multicolumn{3}{|c|}{ RBM3 expression $(n=259)$} \\
\hline & Non-membranous & Membranous & & Low (NS = 0-4) & High (NS = 6-9) & \\
\hline N (\%) & $235(89.7 \%)$ & $27(10.3 \%)$ & $p$-value & $104(40.2 \%)$ & $155(59.8 \%)$ & $p$-value \\
\hline \multicolumn{7}{|l|}{ Age } \\
\hline Mean/median & 71.4/71.4 & $71.2 / 72.4$ & 0.933 & $72.2 / 72.5$ & $70.6 / 70.5$ & 0.069 \\
\hline Range & $51.2-86.6$ & $56.2-86.8$ & & $51.3-86.8$ & $51.2-86.6$ & \\
\hline \multicolumn{7}{|l|}{ Sex } \\
\hline Female & $67(84.8 \%)$ & $12(15.2 \%)$ & 0.088 & $32(40.0 \%)$ & $48(60.0 \%)$ & 0.973 \\
\hline Male & $168(91.8 \%)$ & $15(8.2 \%)$ & & $72(40.2 \%)$ & 107 (59.8\%) & \\
\hline \multicolumn{7}{|l|}{ T-stage } \\
\hline Ta & $113(100.0 \%)$ & $0(0.0 \%)$ & $<0.001$ & $30(26.3 \%)$ & $84(73.7 \%)$ & $<0.001$ \\
\hline $\mathrm{T} 1$ & $73(88.0 \%)$ & $10(12.0 \%)$ & & $28(34.6 \%)$ & $53(65.4 \%)$ & \\
\hline T2-4 & $49(74.2 \%)$ & $17(25.8 \%)$ & & $46(71.9 \%)$ & $18(28.1 \%)$ & \\
\hline \multicolumn{7}{|l|}{ Grade } \\
\hline Low & $128(99.2 \%)$ & $1(0.8 \%)$ & $<0.001$ & $27(20.8 \%)$ & $103(79.2 \%)$ & $<0.001$ \\
\hline High & 107 (80.5\%) & 26 (19.5\%) & & 77 (59.7\%) & $52(40.3 \%)$ & \\
\hline
\end{tabular}

51). The non-classic tumour group included UBC with giant cell carcinoma $(n=2)$, nested $(n=1)$, glandular $(n=5)$, squamous $(n=14)$, trophoblastic $(n=2)$, microcystic $(n=3)$, micropapillary $(n=9)$, plasmacytoid $(n=1)$ and sarcomatoid differentiation $(n=11)$. Kaplan-Meier analysis demonstrated that the 5-year OS differed significantly between groups, with the lowest 5-year OS for the classic group (Additional file 3 ). This was confirmed in univariable Cox regression analysis, with the non-classic tumour group having a significantly reduced survival (HR 8.58, 95\% CI 4.87$15.12, p<0.001)$ and this association remained significant in multivariable analysis (HR 5.87, 95\%CI 1.98-
17.44, $p=0.001)$. As shown in Table 3, non-classic tumour types were significantly correlated with a more advanced higher T-stage $(p<0.001)$, grade $(p<$ 0.001), membranous PODXL expression $(p<0.001)$ and low RBM3 expression $(p<0.001)$.

After adding tumour type as a factor into the multivariable Cox regression analysis of the entire cohort, together with both PODXL and RBM3, RBM3 expression remained significant (HR 1.84, 95\% CI 1.09-3.10, $p=$ 0.022), but not PODXL expression (HR 1.43, 95\% CI $0.77-2.67, p=0.257$ ). Addition of tumour type to the multivariable analyses in Table 2 yielded similar results (data not shown).
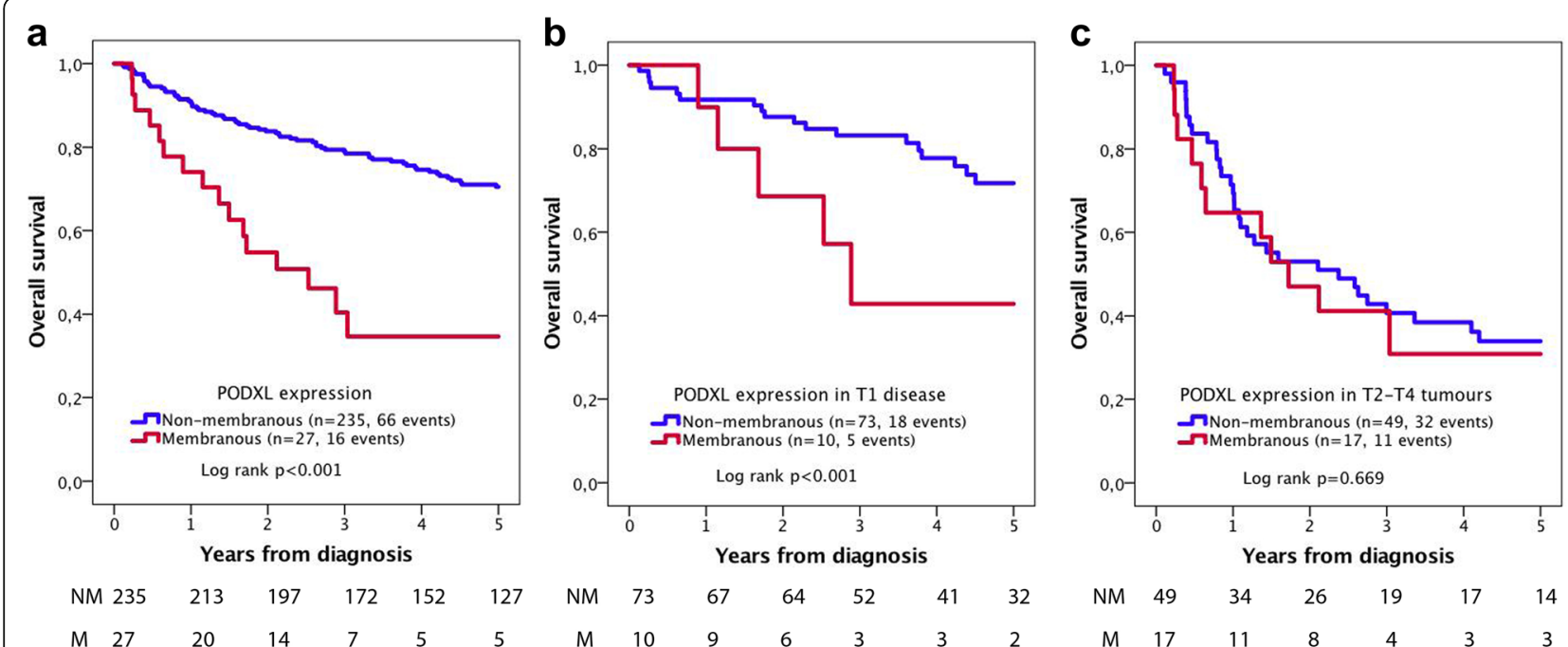

Fig. 3 Five-year overall survival according to PODXL expression. Kaplan-Meier analysis of PODXL expression in relation to 5-year overall survival in (a) the full cohort, (b) T1 tumours and (c) T2-T4 tumours 
Table 2 Relative risk of overall death within 5 years according to clinicopathological factors and expression of PODXL and RBM3

\begin{tabular}{|c|c|c|c|c|c|}
\hline & \multirow[t]{2}{*}{$\mathrm{n}$ (events) } & Univariable & \multirow[t]{2}{*}{$P$-value } & Multivariable & \multirow[t]{2}{*}{$P$-value } \\
\hline & & HR $(95 \% \mathrm{Cl})$ & & HR $(95 \%$ Cl) & \\
\hline \multicolumn{6}{|l|}{ Entire cohort } \\
\hline \multicolumn{6}{|l|}{$\mathrm{Age}^{\mathrm{a}}$} \\
\hline Continuous & $258(79)$ & $1.04(1.01-1.07)$ & 0.010 & $0.96(0.59-1.57)$ & 0.875 \\
\hline \multicolumn{6}{|l|}{$\operatorname{Sex}^{a}$} \\
\hline Female & $79(23)$ & 1.00 & & 1.00 & \\
\hline Male & $179(56)$ & $1.06(0.65-1.72)$ & 0.827 & $1.03(1.00-1.06)$ & 0.078 \\
\hline \multicolumn{6}{|l|}{ Grade $^{a}$} \\
\hline Low & $129(20)$ & 1.00 & & 1.00 & \\
\hline High & $129(59)$ & $3.86(2.32-6.42)$ & $<0.001$ & $1.61(0.82-3.18)$ & 0.165 \\
\hline \multicolumn{6}{|l|}{ Stage $\mathrm{e}^{\mathrm{a}}$} \\
\hline $\mathrm{Ta}$ & $113(16)$ & 1.00 & & 1.00 & \\
\hline $\mathrm{T} 1$ & $81(21)$ & $2.20(1.14-4.21)$ & 0.018 & $1.69(0.81-3.56)$ & 0.165 \\
\hline $\mathrm{T} 2-\mathrm{T} 4$ & $64(42)$ & $8.20(4.58-14.66)$ & $<0.001$ & $4.85(2.23-10.55)$ & $<0.001$ \\
\hline \multicolumn{6}{|l|}{ PODXL expression } \\
\hline Non-membranous & $235(66)$ & 1.00 & & 1.00 & \\
\hline Membranous & $27(16)$ & $3.28(1.89-5.69)$ & $<0.001$ & $1.49(0.83-2.68)$ & 0.181 \\
\hline \multicolumn{6}{|l|}{ RBM3 expression } \\
\hline High & $155(29)$ & 1.00 & & 1.00 & \\
\hline Low & $104(50)$ & $3.19(2.02-5.04)$ & $<0.001$ & $1.85(1.11-3.09)$ & 0.018 \\
\hline \multicolumn{6}{|l|}{ T1 disease } \\
\hline \multicolumn{6}{|l|}{$\mathrm{Age}^{\mathrm{a}}$} \\
\hline Continuous & $81(21)$ & $1.01(0.96-1.07)$ & 0.604 & $1.08(1.00-1.17)$ & 0.066 \\
\hline \multicolumn{6}{|l|}{$\operatorname{Sex}^{\mathrm{a}}$} \\
\hline Female & $21(6)$ & 1.00 & & 1.00 & \\
\hline Male & $60(15)$ & $1.05(0.41-2.72)$ & 0.914 & $0.93(0.36-2.42)$ & 0.884 \\
\hline \multicolumn{6}{|l|}{ Grade $^{a}$} \\
\hline Low & $28(4)$ & 1.00 & & 1.00 & \\
\hline High & $53(17)$ & $2.17(0.73-6.47)$ & 0.163 & $1.63(0.50-6.84)$ & 0.412 \\
\hline \multicolumn{6}{|l|}{ PODXL expression } \\
\hline Non-membranous & $73(18)$ & 1.00 & & 1.00 & \\
\hline Membranous & $10(5)$ & $2.83(1.04-7.72)$ & 0.042 & $2.60(0.91-7.39)$ & 0.073 \\
\hline \multicolumn{6}{|l|}{ RBM3 expression } \\
\hline High & $53(9)$ & 1.00 & & 1.00 & \\
\hline Low & $28(12)$ & $2.64(1.11-6.27)$ & 0.028 & $2.63(1.01-6.84)$ & 0.047 \\
\hline \multicolumn{6}{|l|}{ T2-T4 disease } \\
\hline \multicolumn{6}{|l|}{$\mathrm{Age}^{\mathrm{a}}$} \\
\hline Continuous & $64(42)$ & $1.00(0.96-1.04)$ & 0.935 & $1.08(1.00-1.17)$ & 0.066 \\
\hline \multicolumn{6}{|l|}{$\operatorname{Sex}^{\mathrm{a}}$} \\
\hline Female & $18(12)$ & 1.00 & & 1.00 & \\
\hline Male & $46(30)$ & $0.77(0.39-1.52)$ & 0.453 & $0.93(0.36-2.42)$ & 0.884 \\
\hline \multicolumn{6}{|l|}{ Grade $^{a}$} \\
\hline Low & $4(2)$ & 1.00 & & 1.00 & \\
\hline High & $60(40)$ & $1.79(0.43-7.44)$ & 0.424 & $1.63(0.51-5.25)$ & 0.412 \\
\hline
\end{tabular}


Table 2 Relative risk of overall death within 5 years according to clinicopathological factors and expression of PODXL and RBM3 (Continued)

\begin{tabular}{|c|c|c|c|c|c|}
\hline \multicolumn{6}{|l|}{ PODXL expression } \\
\hline Non-membranous & $47(31)$ & 1.00 & & 1.00 & \\
\hline Membranous & $17(11)$ & $1.16(0.54-2.31)$ & 0.669 & $1.08(0.53-2.20)$ & 0.832 \\
\hline \multicolumn{6}{|l|}{ RBM3 expression } \\
\hline High & $18(9)$ & 1.00 & & 1.00 & \\
\hline Low & $46(33)$ & $2.01(0.95-4.23)$ & 0.066 & $2.22(0.94-5.28)$ & 0.071 \\
\hline
\end{tabular}

${ }^{a}$ Cases included in the univariable analysis of clinicopathological factors were those that had information on both PODXL and RBM3 expression

\section{Discussion}

This study examined the prognostic impact of PODXL and RBM3 expression in tumours from incident cases of UBC in a large, population-based cohort. The results provide further evidence of low RBM3 expression being associated with unfavourable clinicopathological characteristics and an independent factor of decreased survival, and of PODXL expression being associated with more aggressive tumour characteristics and reduced survival, however not independently of established prognostic factors.

PODXL is a protein that in experimental studies is associated with epithelial mesenchymal transition (EMT) and, hence, the invasion and spread of tumours [29]. High expression of PODXL, in particular in the membrane of tumour cells, has previously been demonstrated to correlate to an impaired prognosis in many major cancer forms like breast cancer [9], colorectal cancer [10-12], ovarian cancer [13], glioblastoma [14] and urothelial bladder cancer [15]. The results from this study further validate that membranous expression of PODXL is correlated to more aggressive tumours as it could not be demonstrated in non-invasive Ta-tumours. In addition, it was significantly associated with a reduced survival in univariable analysis, in the full cohort and in $\mathrm{T} 1$ disease, but not in T2-T4 disease. The latter may perhaps reflect the fact that more aggressive and unstable tumours reside in the MI group, therefore diluting the prognostic impact of PODXL expression in the NMI group. Although this study failed to demonstrate a significant association between PODXL expression and survival in multivariable analysis, it should be pointed out that, apart from T-stage, that was an independent prognostic factor in the entire cohort, no other factors provided an independent prognostic value. PODXL expression was borderline significantly associated with a reduced survival in multivariable analysis in the T1 group, which is in line with previous findings [15]. Hence, the prognostic value of PODXL appears to be most evident in T1 disease, and its expression may possibly add value to current prognostic models.

Herein, a significantly decreased 5-year OS was demonstrated for patients with tumours displaying low expression of RBM3, which is well in line with previous findings in UBC [26], as well as breast cancer [17], epithelial ovarian cancer [18, 30], prostate cancer $[19,21]$, testicular cancer [25], oesophageal and gastric cancer [23] and melanoma [24].
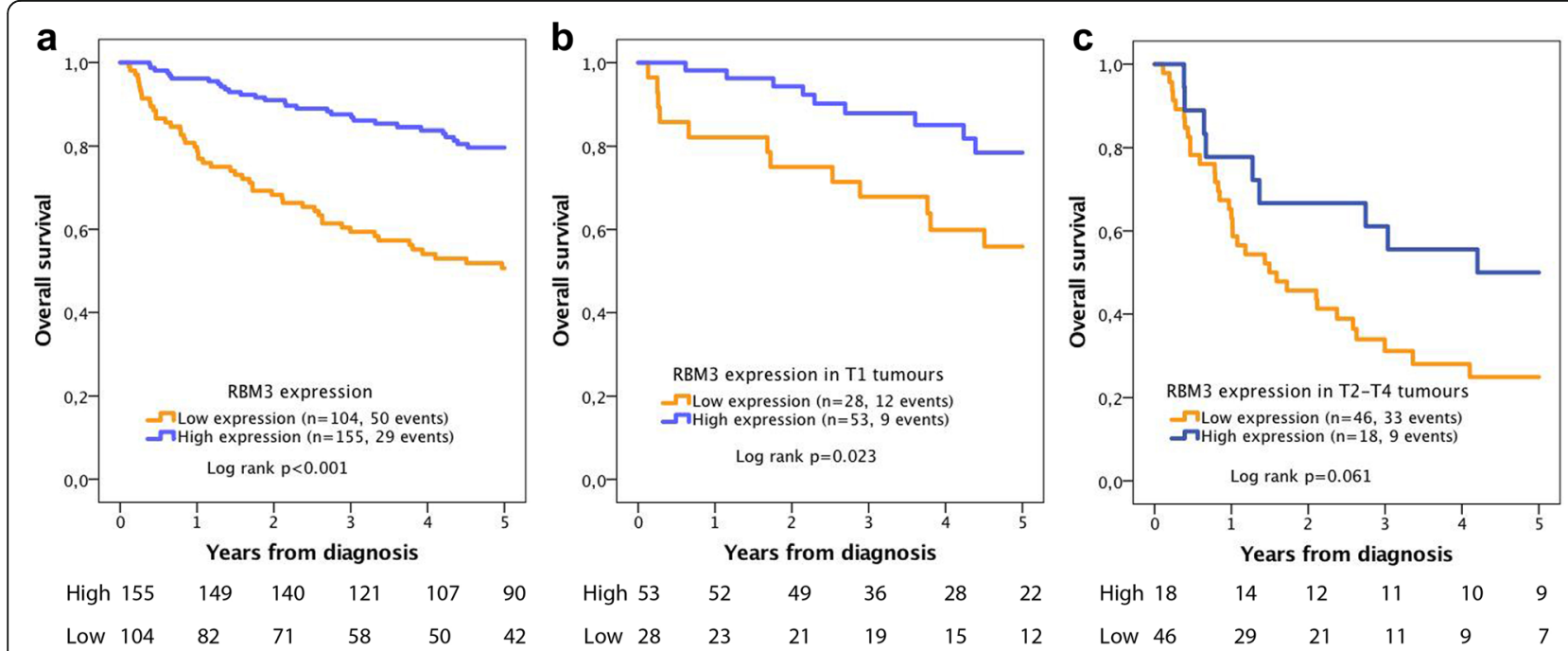

$\begin{array}{llllll}\text { High } 155 & 149 & 140 & 121 & 107 & 90\end{array}$

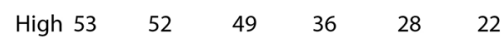

Low 46

Fig. 4 Five-year overall survival according to RBM3 expression. Kaplan-Meier analysis of RBM3 expression in relation to 5-year overall survival in (a) the full cohort, (b) T1 tumours and (c) T2-T4 tumours 
Table 3 Associations between RBM3 and PODXL expression and tumour type

\begin{tabular}{|c|c|c|c|c|c|c|c|}
\hline \multicolumn{4}{|l|}{ PODXL expression } & \multicolumn{4}{|l|}{ RBM3 expression } \\
\hline & Non-membranous & Membranous & & & Low (NS =0-4) & High (NS =6-9) & \\
\hline Tumour type & & & & Tumour type & & & \\
\hline $\begin{array}{l}\text { Non-invasive low grade } \\
(n=97)\end{array}$ & 97 (100\%) & $0(0 \%)$ & $p<0.001$ & $\begin{array}{l}\text { Non-invasive low grade } \\
(n=98)\end{array}$ & $25(25.5 \%)$ & $73(74.5 \%)$ & $p<0.001$ \\
\hline $\begin{array}{l}\text { Non-invasive high grade } \\
(n=16)\end{array}$ & $16(100 \%)$ & $0(0 \%)$ & & $\begin{array}{l}\text { Non-invasive high grade } \\
(n=16)\end{array}$ & $5(31.3 \%)$ & $11(68.7 \%)$ & \\
\hline $\begin{array}{l}\text { Invasive classic UBC } \\
(n=99)\end{array}$ & 85 (85.9\%) & $14(14.1 \%)$ & & $\begin{array}{l}\text { Invasive classic UBC } \\
(n=99)\end{array}$ & $41(42,3 \%)$ & $54(57.7 \%)$ & \\
\hline $\begin{array}{l}\text { Invasive non-classic UBC } \\
(n=50)\end{array}$ & $37(74.0 \%)$ & $13(26.0 \%)$ & & $\begin{array}{l}\text { Invasive non-classic UBC } \\
(n=48)\end{array}$ & $33(68.8 \%)$ & $15(31.2 \%)$ & \\
\hline
\end{tabular}

On a cellular level, RBM3 is normally expressed when cells are exposed to conditions such as hypoxia and cell starvation. These conditions are present in the environment of a malignant tumour [31]. The role of RBM3 in adverse growth conditions is to increase cell survival by means of hindering the normal decrease of protein synthesis and promoting cells to continue through the cell cycle [16, 32]. It has the making of a powerful oncoprotein, but is linked to an improved survival. Perhaps this reflects the regulatory role of its binding to RNA and modulation of RNA expression, making cells more genetically stable, or to the correlation between downregulation of RBM3 and reduced sensitivity to cisplatin in epithelial ovarian cancer in vitro [18], presumably by processes involved in DNA integrity and repair [30]. The indication that RBM3 may be a predictive marker for cisplatin response is in line with the observed association between low RBM3 expression and treatment failure in non-seminatous testicular germ cell cancer [25], in which cisplatin treatment is highly effective. Cisplatin is frequently used for neoadjuvant treatment in MI and palliative treatment in metastatic UBC [4]. Therefore, it would be of interest to study the association of RBM3 with cisplatin sensitivity in this type of cancer.

The T1 category is a subgroup with an urgent need of prognostic biomarkers, as they have a non-negligible risk for progression to muscle-invasive stages [33]. Interestingly, low RBM3 expression was the only significant independent marker of poor prognosis in the T1 group. Therefore, RBM3 may be a clinically useful marker to select patients with high risk of muscle-invasive disease, and, hence, in need of early cystectomy.

Non-classical differentiation was associated with low RBM3 expression, membranous PODXL expression and an adverse outcome for patients. The tumour types included in this group are known to have a poor prognosis compared to classic UBC, apart from microcystic differentiation, for which the clinical relevance is unknown $[34,35]$. The association of non-classical tumours with poor prognosis was validated in our study, however, these subtypes are rare and, compared to the prognostic impact of the two herein examined biomarkers, of limited clinical value to the UBC patient population as a whole.

The herein used antibody for detection of RBM expression has been extensively validated [20,30] and the only seeming contradiction to our findings regarding the prognostic value of RBM3 expression in UBC is a TMA study on cystectomy specimens [36]. However, clinical follow-up was only available for 106 patients out of a total reported number of 274 cases reported in this study, with a mean duration of 2 years. The data presented did not meet the reporting recommendations for tumour marker prognostic studies (REMARK) criteria [37] regarding neither patient characterization, the reporting of data, nor the analysis and presentation. The difference in results between this study and ours may also be due to the fact that the most evident prognostic value of RBM3 expression seems to be for the NMI tumours. In terms of clinical application, this is certainly beneficial. The treatment of MI tumours is cystectomy if the patient is fit for surgery, but the difficulty in choosing therapies lies more in the NMI group.

\section{Conclusions}

The results from this study validate the prognostic value of PODXL and RBM3 expression in UBC, with RBM3 being the potentially most clinically useful biomarker for prognostic stratification of patients with $\mathrm{T} 1$ disease. These findings merit further study and validation also in a prospective setting.

\section{Additional files}

Additional file 1: Kaplan-Meier analysis of 5-year overall survival according to all nuclear scores of RBM3 expression. (JPG $33 \mathrm{~kb}$ )

Additional file 2: Specified non-classical tumour types and their relation to PODXL and RBM3 expression. (DOCX $90 \mathrm{~kb}$ )

Additional file 3: Kaplan-Meier analysis of 5-year overall survival according to tumour type. (JPG $764 \mathrm{~kb}$ ) 


\section{Abbreviations}

MDCS: Malmö Diet and Cancer study; MI: Muscle invasive; NMI: Non-muscle invasive; NS: Nuclear score; PODXL: Podocalyxin like protein; RBM3: RNAbinding motif 3; TMA: Tissue Micro Array; UBC: Urothelial bladder cancer

\section{Acknowledgments}

Not applicable.

\section{Funding}

This work was supported by grants from the Swedish Cancer Society; the Swedish Research Council; the Swedish Government Grant for Clinical Research, the Gunnar Nilsson Cancer Foundation; the Mrs Berta Kamprad Foundation, Lund University Faculty of Medicine and University Hospital Research Grants.

\section{Availability of data and materials}

All data generated or analysed during this study are included in this published article.

\section{Authors' contributions}

$\mathrm{KB}$ evaluated the immunohistochemical staining, performed the statistical analyses and drafted the manuscript. BN constructed the TMAs and performed the immunohistochemical staining. GA and CW collected clinical data. GA helped draft the manuscript. KJ conceived of the study, performed the histopathological re-evaluation, evaluated the immunohistochemistry, and helped draft the manuscript. All authors read and approved the final manuscript.

\section{Competing interests}

$\mathrm{KJ}$ is inventor on patents related to the use of RBM3 and PODXL as prognostic biomarkers in UBC. The other authors declare that they have no competing interests.

\section{Consent for publication}

Not applicable.

\section{Ethics approval and consent to participate}

All EU and national regulations and requirements for handling human samples have been fully complied with during the conduct of this project; i.e. decision no. 1110/94/EC of the European Parliament and of the Council (OJL126 18,5,94), the Helsinki Declaration on ethical principles for medical research involving human subjects, and the EU Council Convention on human rights and Biomedicine. The study was approved of by the Ethics committee of Lund University (ref nr 530/08 and 161/15). Written informed consent has been obtained from each subject at study entry.

\section{Publisher's Note}

Springer Nature remains neutral with regard to jurisdictional claims in published maps and institutional affiliations.

\section{Author details}

${ }^{1}$ Department of Clinical Sciences Lund, Division of Oncology and Pathology, Lund University, Lund, Sweden. ${ }^{2}$ Department of Translational Medicine, Lund University, Malmö, Sweden. ${ }^{3}$ Department of Urology, Skåne University Hospital, Malmö, Sweden.

\section{Received: 27 October 2016 Accepted: 6 March 2017} Published online: 14 March 2017

\section{References}

1. Burger M, Catto JW, Dalbagni G, Grossman HB, Herr H, Karakiewicz P, et al. Epidemiology and risk factors of urothelial bladder cancer. Eur Urol. 2013; 63(2):234-41.

2. Babjuk M, Bohle A, Burger M, Capoun O, Cohen D, Comperat EM, et al. EAU Guidelines on Non-Muscle-invasive Urothelial Carcinoma of the Bladder: Update 2016. Eur Urol. 2017;71(3):447-461.

3. Cambier S, Sylvester RJ, Collette L, Gontero P, Brausi MA, van Andel G, et al. EORTC Nomograms and Risk Groups for Predicting Recurrence, Progression, and Disease-specific and Overall Survival in Non-Muscle-invasive Stage TaT1 Urothelial Bladder Cancer Patients Treated with 1-3 Years of Maintenance Bacillus Calmette-Guerin. Eur Urol. 2016;69(1):60-9.
4. Sternberg CN, Bellmunt J, Sonpavde G, Siefker-Radtke AO, Stadler WM, Bajorin DF, et al. ICUD-EAU International Consultation on Bladder Cancer 2012: Chemotherapy for urothelial carcinoma-neoadjuvant and adjuvant settings. Eur Urol. 2013;63(1):58-66.

5. Breau RH, Karnes RJ, Farmer SA, Thapa P, Cagiannos I, Morash C, et al. Progression to detrusor muscle invasion during urothelial carcinoma surveillance is associated with poor prognosis. BJU Int. 2014;113(6):900-6.

6. Schrier BP, Hollander MP, van Rhijn BW, Kiemeney LA, Witjes JA. Prognosis of muscle-invasive bladder cancer: difference between primary and progressive tumours and implications for therapy. Eur Urol. 2004;45(3):292-6.

7. Takeda T, Go WY, Orlando RA, Farquhar MG. Expression of podocalyxin inhibits cell-cell adhesion and modifies junctional properties in Madin-Darby canine kidney cells. Mol Biol Cell. 2000;11(9):3219-32.

8. Nielsen JS, Graves ML, Chelliah S, Vogl AW, Roskelley CD, McNagny KM. The CD34-related molecule podocalyxin is a potent inducer of microvillus formation. PLoS One. 2007;2(2):e237.

9. Somasiri A, Nielsen JS, Makretsov N, McCoy ML, Prentice L, Gilks CB, et al. Overexpression of the anti-adhesin podocalyxin is an independent predictor of breast cancer progression. Cancer Res. 2004;64(15):5068-73.

10. Larsson A, Fridberg M, Gaber A, Nodin B, Leveen P, Jonsson G, et al. Validation of podocalyxin-like protein as a biomarker of poor prognosis in colorectal cancer. BMC Cancer. 2012;12:282.

11. Larsson A, Johansson ME, Wangefjord S, Gaber A, Nodin B, Kucharzewska P, et al. Overexpression of podocalyxin-like protein is an independent factor of poor prognosis in colorectal cancer. Br J Cancer. 2011;105(5):666-72.

12. Larsson AH, Nodin B, Syk I, Palmquist I, Uhlen M, Eberhard J, et al. Podocalyxin-like protein expression in primary colorectal cancer and synchronous lymph node metastases. Diagn Pathol. 2013;8:109.

13. Cipollone JA, Graves ML, Kobel M, Kalloger SE, Poon T, Gilks CB, et al. The anti-adhesive mucin podocalyxin may help initiate the transperitoneal metastasis of high grade serous ovarian carcinoma. Clin Exp Metastasis. 2012:29(3):239-52.

14. Binder ZA, Siu IM, Eberhart CG, Ap Rhys C, Bai RY, Staedtke V, et al. Podocalyxin-like protein is expressed in glioblastoma multiforme stem-like cells and is associated with poor outcome. PLoS One. 2013;8(10):e75945.

15. Boman $K$, Larsson AH, Segersten U, Kuteeva $E$, Johannesson H, Nodin B, et al. Membranous expression of podocalyxin-like protein is an independent factor of poor prognosis in urothelial bladder cancer. Br J Cancer. 2013: 108(11):2321-8

16. Sureban SM, Ramalingam S, Natarajan G, May R, Subramaniam D, Bishnupuri $\mathrm{KS}$, et al. Translation regulatory factor RBM3 is a proto-oncogene that prevents mitotic catastrophe. Oncogene. 2008;27(33):4544-56.

17. Jogi A, Brennan DJ, Ryden L, Magnusson K, Ferno M, Stal O, et al. Nuclear expression of the RNA-binding protein RBM3 is associated with an improved clinical outcome in breast cancer. Mod Pathol. 2009:22(12):1564-74.

18. Ehlen A, Brennan DJ, Nodin B, O'Connor DP, Eberhard J, AlvaradoKristensson $\mathrm{M}$, et al. Expression of the RNA-binding protein RBM3 is associated with a favourable prognosis and cisplatin sensitivity in epithelial ovarian cancer. J Transl Med. 2010:8:78.

19. Jonsson L, Gaber A, Ulmert D, Uhlen M, Bjartell A, Jirstrom K. High RBM3 expression in prostate cancer independently predicts a reduced risk of biochemical recurrence and disease progression. Diagn Pathol. 2011;6:91.

20. Hjelm B, Brennan DJ, Zendehrokh N, Eberhard J, Nodin B, Gaber A, et al. High nuclear RBM3 expression is associated with an improved prognosis in colorectal cancer. Proteomics Clin Appl. 2011;5(11-12):624-35.

21. Zeng Y, Wodzenski D, Gao D, Shiraishi T, Terada N, Li Y, et al. Stress-response protein RBM3 attenuates the stem-like properties of prostate cancer cells by interfering with CD44 variant splicing. Cancer Res. 2013;73(13):4123-33.

22. Nodin B, Fridberg M, Jonsson L, Bergman J, Uhlen M, Jirstrom K. High MCM3 expression is an independent biomarker of poor prognosis and correlates with reduced RBM3 expression in a prospective cohort of malignant melanoma. Diagn Pathol. 2012;7:82

23. Jonsson L, Hedner C, Gaber A, Korkocic D, Nodin B, Uhlen M, et al. High expression of RNA-binding motif protein 3 in esophageal and gastric adenocarcinoma correlates with intestinal metaplasia-associated tumours and independently predicts a reduced risk of recurrence and death. Biomarker Res. 2014;2:11

24. Jonsson L, Bergman J, Nodin B, Manjer J, Ponten F, Uhlen M, et al. Low RBM3 protein expression correlates with tumour progression and poor prognosis in malignant melanoma: an analysis of 215 cases from the Malmo Diet and Cancer Study. J Transl Med. 2011;9:114. 
25. Olofsson SE, Nodin B, Gaber A, Eberhard J, Uhlen M, Jirstrom K, et al. Low RBM3 protein expression correlates with clinical stage, prognostic classification and increased risk of treatment failure in testicular nonseminomatous germ cell cancer. PLoS One. 2015;10(3):e0121300.

26. Boman K, Segersten U, Ahlgren G, Eberhard J, Uhlen M, Jirstrom K, et al. Decreased expression of RNA-binding motif protein 3 correlates with tumour progression and poor prognosis in urothelial bladder cancer. BMC Urol. 2013;13:17

27. Manjer J, Carlsson S, Elmstahl S, Gullberg B, Janzon L, Lindstrom M, et al. The Malmo Diet and Cancer Study: representativity, cancer incidence and mortality in participants and non-participants. Eur J Cancer Prev. 2001;10(6): 489-99

28. Wennersten C, Andersson G, Boman K, Nodin B, Gaber A, Jirstrom K. Incident urothelial cancer in the Malmo Diet and Cancer Study: cohort characteristics and further validation of ezrin as a prognostic biomarker. Diagn Pathol. 2014;9:189.

29. Meng X, Ezzati P, Wilkins JA. Requirement of podocalyxin in TGF-beta induced epithelial mesenchymal transition. PLoS One. 2011;6(4):e18715.

30. Ehlen A, Nodin B, Rexhepaj E, Brandstedt J, Uhlen M, Alvarado-Kristensson $M$, et al. RBM3-regulated genes promote DNA integrity and affect clinical outcome in epithelial ovarian cancer. Transl Oncol. 2011;4(4):212-21.

31. Nakazawa MS, Keith B, Simon MC. Oxygen availability and metabolic adaptations. Nat Rev Cancer. 2016;16(10):663-73.

32. Matsuda A, Ogawa M, Yanai H, Naka D, Goto A, Ao T, et al. Generation of mice deficient in RNA-binding motif protein 3 (RBM3) and characterization of its role in innate immune responses and cell growth. Biochem Biophys Res Commun. 2011;411(1):7-13.

33. Kurth KH, Denis L, Bouffioux C, Sylvester R, Debruyne FM, Pavone-Macaluso $M$, et al. Factors affecting recurrence and progression in superficial bladder tumours. Eur J Cancer. 1995;31A(11):1840-6.

34. Amin MB. Histological variants of urothelial carcinoma: diagnostic, therapeutic and prognostic implications. Mod Pathol. 2009;22 Suppl 2:S96-S118.

35. Venyo AK. Microcystic variant of urothelial carcinoma. Adv Urol. 2013;2013: 654751

36. Florianova L, Xu B, Traboulsi S, Elmansi H, Tanquay S, Aprikian A, et al. Evaluation of RNA-binding motif protein 3 expression in urothelial carcinoma of the bladder: an immunohistochemical study. World J Surg Oncol. 2015;13:317.

37. Altman DG, McShane LM, Sauerbrei W, Taube SE. Reporting Recommendations for Tumor Marker Prognostic Studies (REMARK): explanation and elaboration. PLoS Med. 2012;9(5):e1001216.

\section{Submit your next manuscript to BioMed Central and we will help you at every step:}

- We accept pre-submission inquiries

- Our selector tool helps you to find the most relevant journal

- We provide round the clock customer support

- Convenient online submission

- Thorough peer review

- Inclusion in PubMed and all major indexing services

- Maximum visibility for your research

Submit your manuscript at www.biomedcentral.com/submit 